\title{
Multi-sensor Fusion Workflow for Accurate Classification and Mapping of Sugarcane Crops
}

\author{
Marife Kung Villareal \\ School of Engineering, University of San Carlos Talamban \\ Campus, Cebu City, Philippines and \\ College of Engineering and Design, Silliman University, \\ Dumaguete City, Philippines \\ mkvillarea127@gmail.com
}

\author{
Alejandro Fernandez Tongco \\ School of Engineering, \\ University of San Carlos, \\ Cebu City, Philippines \\ aftongco@usc.edu.ph
}

\begin{abstract}
This study aims to assess the classification accuracy of a novel mapping workflow for sugarcane crops identification that combines light detection and ranging (LiDAR) point clouds and remotely-sensed orthoimages. The combined input data of plant height LiDAR point clouds and multispectral orthoimages were processed using a technique called object-based image analysis (OBIA). The use of multi-source inputs makes the mapping workflow unique and is expected to yield higher accuracy compared to the existing techniques. The multi-source inputs are passed through five phases: data collection, data fusion, image segmentation, accuracy validation, and mapping. Data regarding sugarcane crops were randomly collected in ten sampling sites in the study area. Five out of the ten sampling sites were designated as training sites and the remaining five as validation sites. Normalized digital surface model (nDSM) was created using the LiDAR data. The nDSM was paired with Orthophoto and segmented for feature extraction in OBIA by developing a ruleset in eCognition software. A rule-set was created to classify and to segment sugarcane using nDSM and Orthophoto from the training and validation area sites. A machine learning algorithm called support vector machine (SVM) was used to classify entities in the image. The SVM was constructed using the nDSM. The height parameter nDSM was applied, and the overall accuracy assessment was $98.74 \%$ with Kappa index agreement (KIA) $\mathbf{9 7 . 4 7 \%}$, while the overall accuracy assessment of sugarcane in the five validation sites were $94.23 \%, 80.28 \%, 94.50 \%, 93.59 \%$, and $93.22 \%$. The results suggest that the mapping workflow of sugarcane crops employing OBIA, LiDAR data, and Orthoimages is attainable. The techniques and process used in this study are potentially useful for the classification and mapping of sugarcane crops.
\end{abstract}

Keywords- image analysis; sugarcane mapping; remote sensing

\section{INTRODUCTION}

Global sugar production is approximately 180 million tons. World production is dominated by Brazil, followed by India, European Union, and Thailand. Sugarcane occupies about $80 \%$ of the world sugar production and is cultivation is concentrated in Asia and South/Central America [1]. Historically, there are recurring supply and demand imbalances reflected in extremely volatile prices on the world market. For the past several decades, world production of sugar has been more than the consumption, leading to low prices and stock overhangs. Due to the result of crop failures in one or more of the main producing countries, there have been shorter periods of deficit. Because of the crop failures short term price spikes, followed by equally sharp declines occur [1]. Increasing attention has been paid to sugarcane plantation in recent years not only for strained sugar supply due to rapid global population increase but also for a growing demand for biomass energy. Considering the global environment, sugarcane is an important resource of alcohol which can be processed into biofuels for motor vehicles and generation of electricity. Regarding economic and environmental aspects, there is a strong demand for effective methods of providing timely and accurate information on sugarcane growing areas and growth conditions at regional and global scale [2]. Despite the contribution of the production of sugarcane to the economic growth in the Philippines, there are insufficient records that can help growers make accurate decisions or proper planning to improve production. According to the Sugar Regulatory Administration (SRA), the sugar industry contributes about Php 87 billion (1.68 billion dollars) to the national economy, with more than 700,000 workers. The productivity contribution of $80 \%$ comes from landholdings with size less than 5 hectares and the $1 \%$ comes from farms greater than 100 hectares [3]. Expanding the production of sugarcane is important to increase yield and maximize profit. The use of remote sensing technology and techniques for detailed mapping and effective data management has facilitated the expansion of small-scale growers and increased agronomic crop yield [4].

Object-based image analysis (OBIA) is gaining much attention in the remote sensing community. It is a subdiscipline of GIScience devoted to partitioning remote sensing (RS) imagery into meaningful image objects. The characteristics of OBIA are assessed through spatial, spectral, and temporal scales [5]. The OBIA workflows are highly customizable, allowing the presence of human semantics and hierarchical networks [6, 7]. Generally, there are two main processes in OBIA: segmentation and classification. Segmentation is the process of grouping adjacent pixels together based on their homogeneity to create meaningful "objects" and these objects are subjected to classification. The segmentation and classification can be processed easily by the different algorithms in eCognition (Trimble eCognition version 
9) because much of the work referred to as OBIA is originated from eCognition software [8-10]. Object-based classification can be done through user-defined rule-sets. Studies have been conducted using OBIA with other remote sensing techniques. Authors in [11-13] combined OBIA with data mining. Authors in $[14,15]$ used OBIA and images from the Landsat/TM/OLI and IRS/LISS-3 satellites. Authors in [16] also used Landsat/TM/OLI for sugarcane mapping, but with MODIS EV12 time series. Authors in [17] used OBIA procedure for UAV images to map and extract information for sugarcane skips in planting row.

The use of OBIA with the nDSM feature from LiDAR data and orthoimages can provide detailed mapping and aid in the precise inventory of agricultural crops. LiDAR is one remote sensing tool used in resource mapping, and it has emerged as a powerful active remote sensing method for direct measurement of 3D plant structure in precision agriculture $[18,19]$. The LiDAR-derived digital elevation information makes it invaluable in improving identification and delineation of remotely-sensed data. LiDAR technology has been applied in several studies concerning crops and geographic features. Authors in [20] used the LiDAR technology to measure sugarcane height. Author in [21] applied LiDAR as a groundbased scanning system for measurements in sugarcane burning operations, whereas authors in $[22,23]$ used it in mangrove extraction in different coastal areas and as one of the layers during data processing. Authors in $[24,25]$ found that height features derived from LiDAR data can significantly differentiate between high and low vegetation. In [26], highresolution digital elevation models (DEM) derived from remotely sensed LiDAR data for contrast mapping used the dissimilarity between artificial and natural slopes to identify and to map discrete features. In [27], LiDAR intensity and height information, contributed to the normalized digital surface model for land-use classification in shady areas. LiDAR-derived agricultural land-cover map and field surveyvalidated data was used for a theoretical biomass resource assessment, as well as an available potential of sugarcane millbased residue in [28]. Multispectral satellite imagery and LiDAR-derived digital elevation information was used for classifying estuarine vegetation types in [29]. OBIA, LiDAR, and orthoimages have been used with several other remote sensing techniques: OBIA can turn remote sensing into a meaningful image object, LiDAR has been used to generate a detailed resource mapping, and RGB with orthoimages visually observe the texture information to produce a combination of the three - a promising technological advancement for resource mapping.

Considering the usefulness of this technique, it is widely studied in agriculture, coastal, and resource mappings, risk management, etc. With the integration of LiDAR data and high spatial resolution orthoimages, more input data layers are available for object-based SVM classification [30, 31]. However, different classes of objects are not separable by direct thresholding. Hence, samples from different classes of objects need to be classified using machine learning algorithms [32]. Among the machine learning algorithms, the SVM is used to classify objects in the image [33]. The SVM represents a group of theoretically superior non-parametric machine learning algorithms where there is no assumption made on the distribution of underlying data [34-36]. The basic concept behind SVM is to search for a balance between the standardized term and the training errors [37]. SVMs are not particular with training sample size. It is possible to successfully work with limited quantity and quality of training samples. In fact, only a quarter of the original training samples in an image are required to produce an equally high accuracy for the classification of two crops [38]. The most important characteristic is SVM's ability to generalize from a limited amount and from the quality of training data [39, 40]. The ruleset developed in eCognition and the validation using SVM for the classification of sugarcane crops is potentially useful to the crop yield estimation of the sugar industry in the Philippines.

Multi-sensor fusion of LiDAR data and orthoimages were employed in the sugarcane plantation in Philippines. The sugar industry requires spatially explicit tools to provide reliable and precise information on sugarcane areas and on the location of sugarcane fields to improve accuracy in monitoring sugarcane production and yield estimates [41-43]. The study aims to assess the classification accuracy of a novel mapping workflow for sugarcane crops identification that combines LiDAR point clouds and remotely-sensed orthoimages. This paper demonstrates the rule-set developed in eCognition using LiDAR data and orthoimages to test and assess accuracy by applying them in the testing and validation sites. SVM is used in the validation sites to obtain accuracy results, which would be beneficial to the Philippines sugar monitoring agency.

\section{MATERIALS AND METHODS}

\section{A. Study Area}

Medellin is a municipality in the northern part of Cebu, Philippines. The study area for this mapping is Barangay Poblacion $\left(11^{\circ} 08^{\prime} 07.0^{\prime \prime} \mathrm{N} 123^{\circ} 57^{\prime} 43^{\prime \prime} \mathrm{E}\right)$, which is one of the villages of Medellin as shown in Figure 1. The main source of income is agriculture, particularly sugarcane crops.

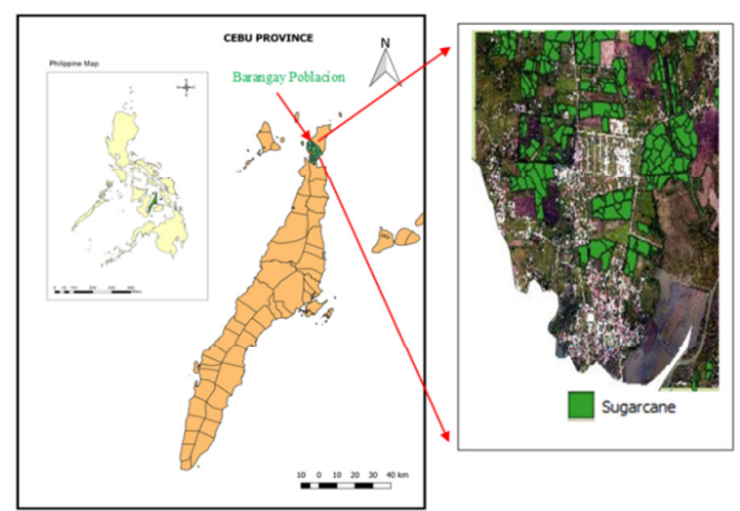

Fig. 1. Map of study area with sugarcane at Barangay Poblacion, Medellin, Cebu Philippines

\section{B. Process Workflow}

This study used OBIA for classification. The process workflow is shown in Figure 2. The five phases in the process 
workflow is data collection, data fusion, image segmentation, accuracy validation, and mapping.

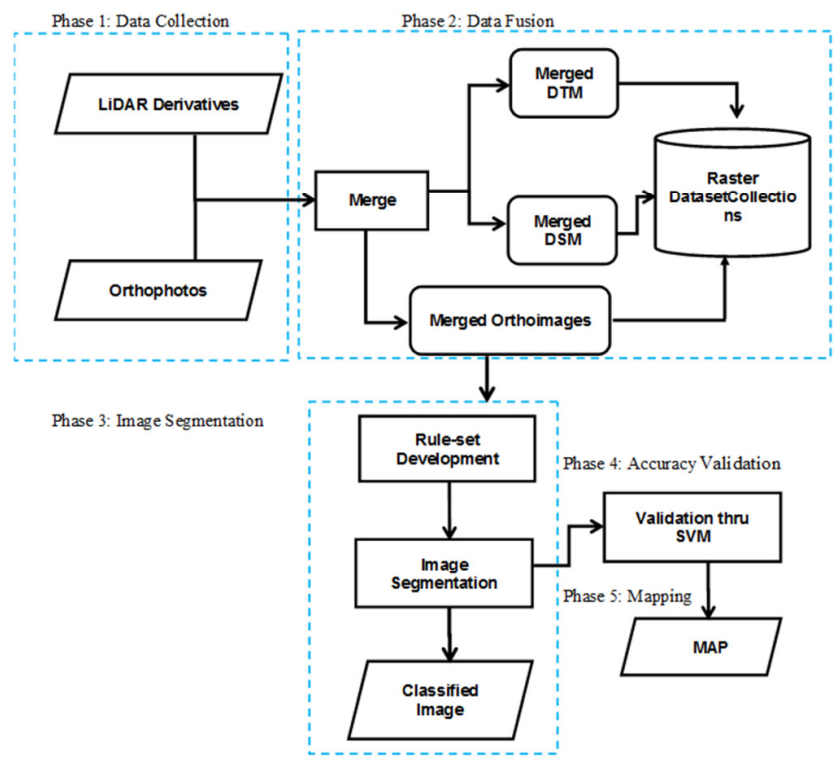

Fig. 2. Process workflow

\section{Data Collection}

LiDAR data and orthoimages used in this study have been pre-processed by Phil-LiDAR Research Center at the University of San Carlos, Talamban-Campus. The LiDAR sensor used was a Pegasus at an altitude of $800 \mathrm{~m}$ and scanning angle of $50^{\circ}$. The resolution was $1 \mathrm{~m}$. Data were collected from the sampling sites via visual interpretation from the orthoimages in the study area. According to [22, 44, 45] samples can be collected after segmentation and checked visually in orthoimages. The nDSM provides the height information of the objects that must be classified. The nDSM was produced by subtracting digital terrain model (DTM) from the canopy surface model or digital surface model (DSM). The orthoimage is an aerial RGB photo. Using the RGB alone, one can perform OBIA (segmentation and classification). The best feature to be used in RGB is texture information which can be observed by visual inspection. According to [46] the classification accuracy and reliability are estimated visually using RGB orthomosaic.

\section{Data Fusion}

The nDSM were integrated with orthoimages for image segmentation and rule-set development in eCognition to produce the classified image. The nDSM provided the real heights of the objects above the ground and was segmented according to a certain height threshold. Thresholding was done on grayscale images. Thresholding an image is classifying these pixels into groups setting an upper and lower bound to each group. The segmentation was done to differentiate highrise objects among others. The nDSM was used from the LiDAR data by applying plant height thresholds. LiDAR data provide accurate measurements of land cover structures in the vertical plane but have limited coverage in the horizontal plane.
RGB data provided extensive coverage of land cover classes in the horizontal plane but were relatively insensitive to variation in their height. Therefore, the integration of LiDAR and multispectral greatly improved the measurement and mapping of landcover classes $[47,48]$. This paper gives emphasis on the classification of a certain class of vegetation which was medium elevation objects with a mean threshold condition of less than or equal to $2.0 \mathrm{~m}$ in the LiDAR nDSM. With RGB alone some object classes like trees can be misclassified as grass or something else. This was where LiDAR data become very useful. The $\mathrm{nDSM}$ was the LiDAR height data normalized with respect to the ground surface. All ground points in nDSM were normalized to zero.

\section{E. Image Segmentation}

Segmentation started with classifying pixels to produce a land cover or land classification result, classification was done on the "segments" or groups of pixels ("object" based classification). There were four image layers used in the objectbased image analysis. These layers were nDSM from LiDAR and orthophoto consisting of three bands, red, green, blue for pre-segmentation process. Image layers were assigned to synchronize the segmentation process. The segmentation process was based on $\mathrm{nDSM}$ and orthophoto images. The features used in RGB were texture information which was observed by visual inspection (Figures 3-4). The rule-set (Figure 5), was developed in eCognition, was used in the five training and the five validation sites in the study area.

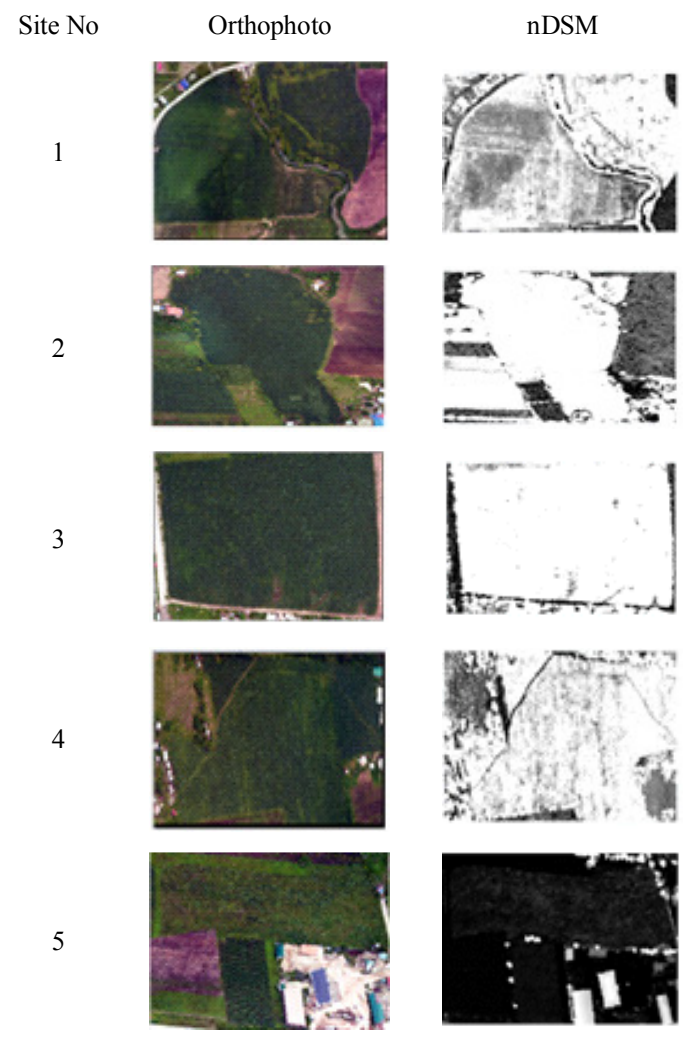

Fig. 3. Training site orthophotos and nDSM of the study area 
Site No
Orthophoto
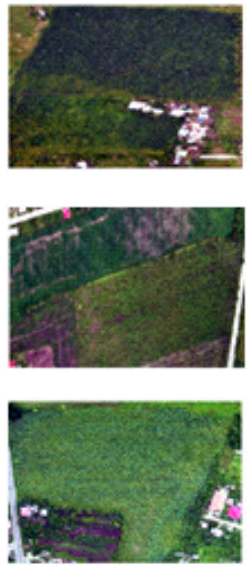

4
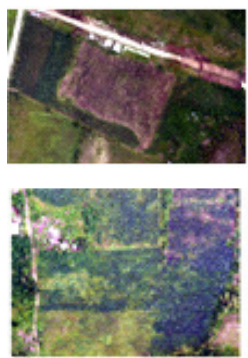

$\mathrm{nDSM}$
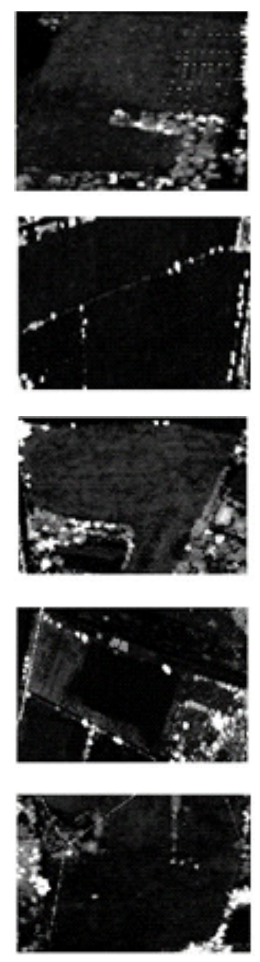

Fig. 4. Validation site orthophotos and nDSM of the study area.

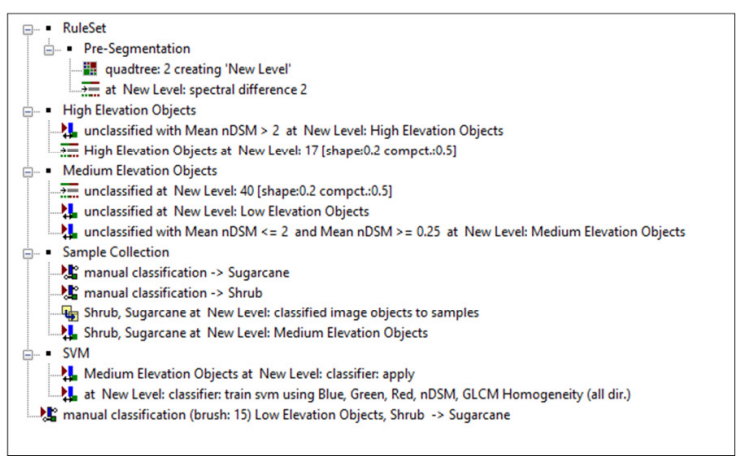

Fig. 5. Rule-set applied to the training and validation sites

Training and validation sites were identified through visual interpretation of the orthoimages. According to [49], the training sites can be interpreted from high resolution imagery. In the segmentation process (Figure 6), the high, medium and low elevation objects were classified.

The quad tree-based was the first segmentation process using nDSM and having a scale parameter of $2.0 \mathrm{~m}$. Quadtree segmentation was used to create image objects by cutting and creating squares of different sizes to easily separate images with significant proportion of background. Scale parameters values were based on a trial and error principle and a suitable value was found through a series of tests [50]. Spectral difference segmentation was conducted with maximum spectral difference of $2.0 \mathrm{~m}$. The spectral difference was to merge neighboring image objects if the difference between their layer mean intensities was below the value given by the maximum spectral difference. Spectral difference was designed to refine existing segmentation results, by merging spectrally similar image objects produced by previous segmentations. The multiresolution segmentation algorithm consecutively merged pixels or existing image objects. Multi-resolution was an optimization procedure which, for a given number of image objects, minimizes the average heterogeneity and maximizes their respective homogeneity. In the pre-classification process, high elevation objects were assigned to a mean $\mathrm{nDSM}$ greater than $2.0(\mathrm{nDSM}>2.0)$. Medium elevation was assigned to mean $\mathrm{nDSM}$ such as $0.25 \leq \mathrm{nDSM} \leq 2.0$. The objects identified for medium elevation were sugarcane and shrub. The "Shrub" class consisted of vegetation or crops that were within the height range of 0.25 and 2 meters. A method needs to be developed on this kind of vegetation. The remaining objects, less than or equal to $0.25 \mathrm{~m}$ were considered as low elevation objects. After assigning objects, another segmentation process follows, the multi-resolution segmentation. In Figure 7, examples of quadtree, spectral difference and multi-resolution segmentation are shown.

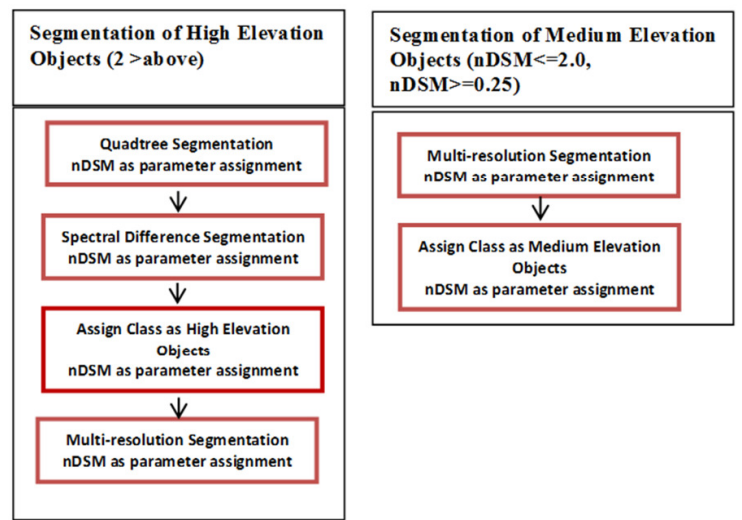

Fig. 6. Segmentation process of high and medium elevation objects.

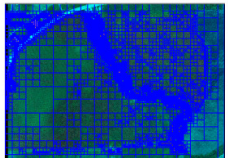

(a)

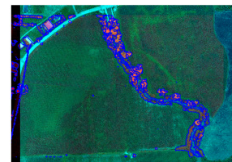

(b)

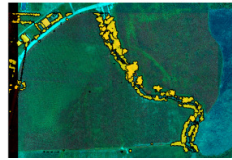

(c)
Fig. 7. Examples of (a) quadtree, (b) spectral difference, and (c) multiresolution segmentations.

Segmentation algorithms were used to subdivide entire images at pixel level, or specific image objects from other domains into smaller image objects. To discriminate other objects, nDSM was used as a discriminating factor to group the objects according to their heights. The orthoimages (RGB) for the texture information of the image were done by visual inspection.

\section{F. Accuracy Validation Using SVM}

SVM is a supervised learning algorithm used to classify entities in an image. It was used to classify land features in the images. The classified objects were obtained from the 
segmentation procedure. nDSM was used as a feature to develop the SVM model. The process was done by sample collection through manual classification. Sugarcane was extracted from the segmented images. The samples collected for sugarcane were 5846. Only a quarter of the original training samples in an image were required to produce equally high classification accuracy. The ability of SVM to generalize well from a limited amount and/or quality of training data is its most important characteristic [39, 40]. SVM was applied to the validation sites to test the accuracy of the samples.

\section{G. Classification Map}

The final classification map was generated using the QGIS software (QGIS ver. 2.18 Las Palmas de G.C.). Segmented images from eCognition were exported to QGIS as raster files and converted to polygons to generate the classified map for Barangay Poblacion. Sugarcane, shrub, high, and low elevation objects were identified.

\section{RESULTS AND DISCUSSION}

\section{A. Classified Images}

Sugarcane crops were extracted and classified using nDSM to discriminate and group objects according to their height. Multi-resolution segmentation in eCognition was used to delineate medium elevation objects using the setting (scale parameter $=40$, shape $=0.2$ and compactness $=0.5$ ). Sugarcane was identified as a medium elevation object based on nDSM.

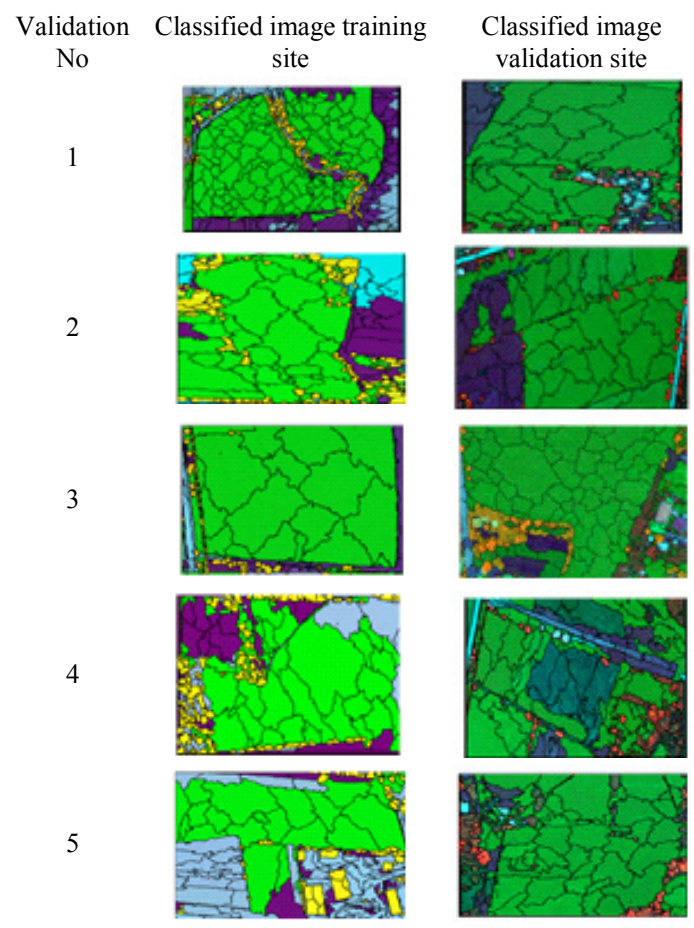

Fig. 8. Classification results on training and validation sites

Then, samples were collected for sugarcane. Orthoimages were used for texture and visual inspection to verify sugarcane crops in the study area. As a result, in the classification process using the SVMs applied to the training and validation sites, sugarcane was identified as shown in Figure 8. A final classified map with sugarcane crops of Barangay Poblacion is shown in Figure 9.

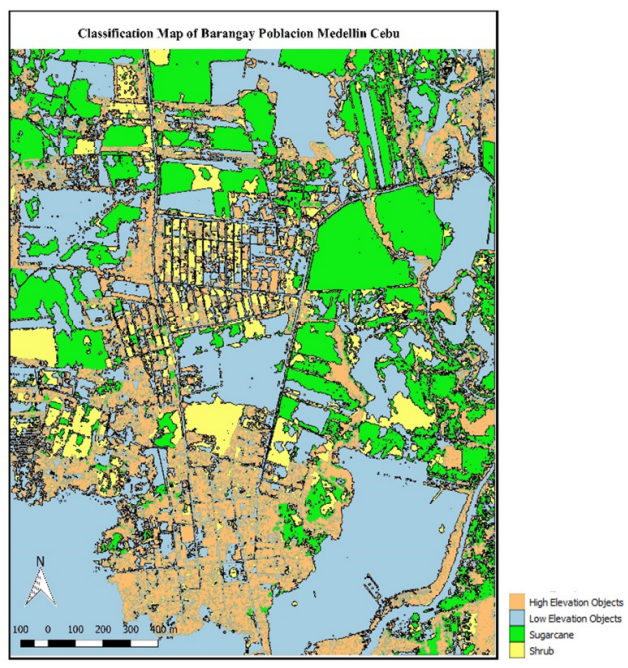

Fig. 9. Land cover classification map of Barangay Poblacion.

\section{B. Accuracy Assessment}

Overall accuracy and KIA for sugarcane are $98.74 \%$ and $97.47 \%$ respectively. Accuracy results were obtained after SVM using eCognition. Table I is an example of the confusion matrix. Accuracy assessment and KIA for each validation site are shown in Table II.

TABLE I. ACCURACY ASSESSMENT AFTER SVM.

\begin{tabular}{|c|c|c|c|}
\hline \multicolumn{4}{|c|}{ Confusion Matrix } \\
\hline User class/Sample & Sugarcane & Shrub & Sum \\
\hline Sugarcane & 5846 & 109 & 5955 \\
\hline Shrub & 30 & 5043 & 5073 \\
\hline Sum & 5876 & 5152 & \\
\hline Accuracy & & & \\
\hline Producer & 0.9948945 & 0.978843 & \\
\hline User & 0.9816961 & 0.994 & \\
\hline Hellden & 0.9882512 & 0.986406 & \\
\hline Short & 0.9767753 & 0.973176 & \\
\hline KIA per class & 0.989 & 0.96082 & \\
\hline Totals & & & \\
\hline Overall Accuracy & 0.9873957 & & \\
\hline KIA & 0.9746584 & & \\
\hline
\end{tabular}

Accuracy and KIA results in validation site 2 were low compared to the other sites due to the area coverage and misclassifications of sugarcane class as shrub class. The similarities in spectral characteristics of sugarcane crops with other vegetation such as shrub, result to low accuracy [29]. Experimenting on other LiDAR derivatives was needed to improve classification and segmentation [22]. The overall accuracy assessment for each validation site shows that sugarcane is correctly classified. According to [51], the Kappa statistic or KIA indicates the extent to which the classification result is better than pure chance: the higher the KIA value, the greater the classification accuracy. With the overall accuracy results, this study will be able to address the existing problem 
of SRA and provide accurate information for policy makers in the sugar industry.

TABLE II. ACCURACY ASSESSMENT AFTER SVM FOR EACH VALIDATION SITE.

\begin{tabular}{|c|c|c|}
\hline Validation Site & Accuracy assessment (\%) & KIA (\%) \\
\hline 1 & 94.23 & 78.91 \\
\hline 2 & 82.76 & 58.63 \\
\hline 3 & 94.50 & 71.90 \\
\hline 4 & 93.59 & 85.46 \\
\hline 5 & 93.22 & 67.67 \\
\hline
\end{tabular}

\section{Assessment of the System Workflow}

The graph of the different overall accuracy assessment, KIA and accuracy for sugarcane in the five validation sites is shown in Figure 10.

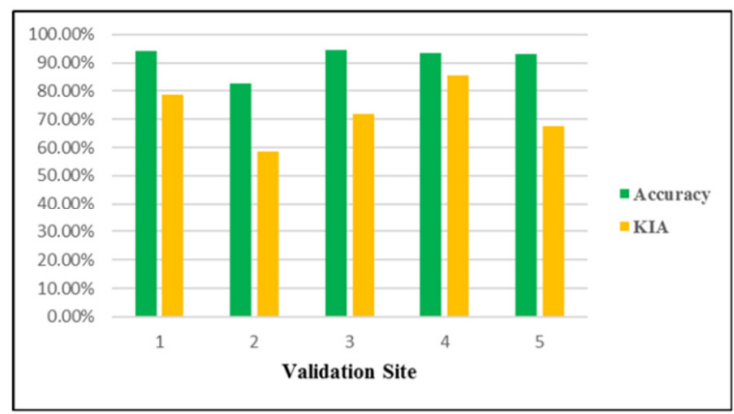

Fig. 10. Graph of sugarcane accuracy assessment and KIA.

The high accuracy of the results indicates that the process workflow developed in this study is applicable and useful in the classification of sugarcane crops in other areas. The overall high accuracy is comparable with using traditional data and techniques [22]. This study extracts sugarcane crops in Barangay Poblacion Medellin Cebu. The high accuracy results of the developed process can be used for mapping and monitoring of sugarcane crops in Philippines and in other sugar producing countries.

\section{CONCLUSION}

This study developed a mapping workflow to assess classification accuracy for sugarcane crop identification using OBIA, LiDAR data and orthoimages. The accuracy results in the validation sites were $94.23 \%, 80.28 \%, 94.50 \%, 93.59 \%$ and $93.22 \%$, and the overall accuracy result was $98.74 \%$ (KIA was $97.47 \%$ ). Therefore, using this workflow in identifying sugarcane in other areas with acceptable accuracy assessment is possible. This can contribute to regional and global scale of providing information on sugarcane growing areas and growth conditions. Further research with additional samples is necessary in order to improve the workflow. The data used in the process are essential because the workflow is dependent on the images provided by LiDAR or any other remote sensing technology.

\section{ACKNOWLEDGEMENT}

Authors wish to thank Phil-LiDAR Research Center at the University of San Carlos, Talamban Campus for the provided data, and Silliman University for the financial support.

\section{REFERENCES}

[1] Canadian Sugar Institute, Global Sugar Trade (WTO), available at: https://sugar.ca/International-Trade/Global-Sugar-Trade-(WTO).aspx

2] L. Hui, J. Chen, Z. Pei, S. Zhang, X. Hu, "Monitoring Sugarcane Growth Using ENVISAT ASAR Data", IEEE Transactions on Geoscience and Remote Sensing, Vol. 47, No. 8, pp. 2572-2580, 2009

[3] Bureau of Agricultural Statistics, 23rd Annual Publication, 2012

[4] E. M. Abdel-Rahman, F. B. Ahmed, "The application of remote sensing techniques to sugarcan (Saccharum spp. hybrid) production: A review of the literature", International Journal of Remote Sensing, Vol. 29, No. 13, pp. 3753-3767, 2008

[5] G. J. Hay, G. Castilla, "Object-based Image Analysis: Strengths, Weaknesses, Opportunities and Threats (SWOT)", in: ISPRS Archives, Vol. 36, 2006

[6] T. Blaschke, K. Johansen, D. Tiede, "Object-Based Image Analysis for Vegetation Mapping and Monitoring", Advances in Environmental Remote Sensing: Sensors Algorithms and Applications, pp. 241-271, CRC Press Taylor \& Francis Group, 2011

[7] M. V. Japitana, J. E. D. Cubillas, A. G. Apdohan, "Coupling LiDAR Data and LANDSAT 8 OLI in Delineating Corn Plantations in Butuan City, Philippines", 36th Asian Conference on Remote Sensing, Quezon City, Metro Manila, Philippines, October 19-23, 2015

[8] M. Baatz, M. Schape, "Multiresolution segmentation - An optimization approach for high quality multi-scale image", Angewandte Geographische Informations, Vol. 12, pp. 12-23, 2000

[9] D. Flanders, M. Hall-Beyer, J. Pereverzoff, "Preliminary evaluation of eCognition object-based software for cut block delineation and feature extraction", Canadian Journal of Remote Sensing, Vol. 29, No. 4, pp. 441-452, 2003

[10] U. C. Benz, G. Hofmann, I. Willhauck, M. Lingenfelder, M. Heynen, "Multi-resolution, object-oriented fuzzy analysis of remote sensing data for GIS-ready information", ISPRS Journal of Photogrammetry and Remote Sensing, Vol. 58, No. 3-4, pp. 239-258, 2004

[11] Z. Zhou, J. Huang, J. Wang, K. Zhang, Z. Kuang, S. Zhong, X. Song, "Object-Oriented Classification of Sugarcane Using Time-Series Middle-Resolution Remote Sensing Data Based on AdaBoost", Plos One, Vol. 10, No. 11, p. e0142069, 2015

[12] A. R. Formaggio, M. A. Vieira, C. D. Renno, D. A. Aguiar, M. P. Mello, "Object-based Image Analysis and Data Mining for Mapping Sugarcane with LANDSAT Imagery in Brazil", The International Archives of the Photogrammetry, Remote Sensing and Spatial Information Sciences, Vol. 38, pp. 553-562, 2012

[13] M. A. Viera, A. Formaggio, C. Renno, C. Atzberger, D. Aguiar, M Mellp, "Object based image analysis and data mining applied to a remotely sensed Landsat time-series to map sugarcane over large areas", Remote Sensing of Environment, Vol. 123, pp. 553-562, 2012

[14] C. J. Cechim, J. A. Johann, J. F. G. Antunes, "Mapping of sugarcane crop area in the Parana State using Landsat/TM/OLI and IRS/LISS-3 Images", Revista Brasileira de Engenharia Agricola e Ambiental, Vol. 21, No. 6, pp. 427-432, 2017

[15] D. Fonseca-Luengo, A. Garcia-Pedrero, M. Lillo-Saavedra, R. Costumero, E. Menasalvas, C. Gonzalo-Martin, "Optimal Scale in a Hierarchical Segmentation Method for Satellite Images", in: Lecture Notes in Computer Science, Vol. 8537, Springer, 2014

[16] C. D. Alves, T. G. Florenzano, D. S. Alves, M. N. Pereira, "Mapping Land Use and Land Cover Changes in a Region of Sugarcane Expansion Using TM and MODIS Data", Revista Brasileira de Cartografia, Vol. 66, No. 2, pp. 337-347, 2014

[17] C. H. W. de Souza, R. A. C. Lamparellib, J. V. Rocha, P. S. G. Magalhaes, "Mapping skips in sugarcane fields using object-based analysis of unmanned aerial vehicle (UAV) images", Computers and Electronics in Agriculture,Vol. 143, pp. 49-56, 2017

[18] W. S. Lee, V. Alchanatis, C. Yang, M. Hirafugi, D. Moshu, C. Li, "Sensing technologies for precision specialty crop production", Computers and Electronics in Agriculture,Vol. 74, No. 1, pp. 2-33, 2010

[19] J. R. Rosell, J. Llorens, R. Sanz, J. Arno, M. Ribes-Dasi, J. Masip, "Obtaining the three-dimensional structure of tree orchards from remote 
2D terestrial LiDAR scanning", Agricultural and Forest Meteorology, Vol. 149, No. 9, pp. 1505-1515, 2009

[20] T. F. Canata, J. P. Molin, A. F. Colaco, R. G. Trevisan, M. Martello, P. R. Fiorio, "Measuring height of sugarcane plants through LiDAR technology", 13th International Conference on Precision Agriculture, Missouri, USA, July 31-August 3, 2016

[21] A. L. Hiscox, "Lidar Measurement Techniques for Understanding Smoke Plume Dynamics in Sugarcane Production", Optical Instrumentation for Energy and Environmental Applications. Optical Society of America, 2013

[22] A. V. Pada, M. A. Silapan, F. Cabanlit, J. Campomanes, J. Garcia "Mangrove Forest Cover Extraction of the Coastal Areas of Negros Occidental, Western Visayas, Philippines Using LiDAR Data", 23th ISPRS Congress, Prague, Chech Republic, July 12-19, 2016

[23] R. V. Peralta, R. L. Jalbuena, C. A. Cruz, A. M. Tamondong, "Development of an Object-based Classification Technique for Extraction of Aquaculture Features using LiDAR and Worldview-2 Satellite Image Data", 13th South East Asian Survey Congress: Expanding the Geospatial Future, Singapore, July 28-31, 2015

[24] A. Charaniya, R. Manduchi, S. Lodha, "Supervised parametric classification of aerial LiDAR data", 2004 Conference on Computer Vision and Pattern Recognition Workshop, Washington, USA, June $27-$ July 2, 2004

[25] Y. Huang, B. Yu, J. Zhou, C. Hu, W. Tan, Z. Hu, “Toward automatic estimation of urban green volume using airborne LiDAR data and highresolution remote sensing images", Frontiers of Earth Science, Vol. 7 , pp. 43-54, 2013

[26] M. D. McCoy, G. P. Asner, M. W. Graves, “Airborne lidar survey of irrigated agricultural landscapes: an application of the slope contrast method", Journal of Archaeological Science, Vol. 38, No. 9, pp. 21412154, 2011

[27] Q. Man, P. Dong, H. Guo, "Pixel- and feature-level fusion of hyperspectral and lidar data for urban land-use classification", International Journal of Remote Sensing, Vol. 36, No. 6, pp. 1618-1644, 2015

[28] M. B. Cadalin, J. Silapan, M. Remolador, M. R. C. Ang, "Biomass Resource Assessment on Theoritical and Available Potential of Sugarcane Using LiDAR-Derived Agricultural Land-Cover Map in Victorias City, Negros Occidental, Philippines", 36th Asian Conference on Remote Sensing, Quezon City, Philippines, October 19-23, 2015

[29] M. Luck-Vogel, C. Mbolambia, K. Rautenbachb, J. Adams, L. Van Niekerkab, "Vegetation mapping in the St Lucia estuary using very high-resolution multispectral imagery and LiDAR", South African Journal of Botany, Vol. 107, pp. 188-199, 2016

[30] L. C. G. David, A. H. Ballado, "Mapping Mangrove Forest from LiDAR Data Using Object- Based Image Analysis and Support Vector Machine: The Case of Calatagan, Batangas", 8th IEEE International Conference Humanoid, Nanotechnology, Information Technology Communication and Control, Environment and Management, Cebu, Philippines, December 9-12, 2015

[31] L. C. G. David, A. H. Ballado, “Object-based land use and land cover mapping from LiDAR data and orthophoto application of decision treebased data selection for SVM classification", IEEE Region 10 Humanitarian Technology Conference, Agra, India, Decemer 21-23, 2016

[32] R. J. Candare, M. V. Japitana, J. E. Cubillas, C. B. Ramirez, "Mapping of High Value Crops Through an Object-Based SVM Model Using LiDAR Data and Orthophoto in Agusan del Norte, Philippines", 23th ISPRS Congress, Prague, Chech Republic, July 12-19, 2016

[33] R. Devadas, R. J. Denham, M. Pringle, "Support Vector Machine Classification of Object-based Data for Crop Mapping, Using Multitemporal Landsat Imagery", International Archives of the Photogrammetry, Remote Sensing and Spatial Information Sciences, Vol. 39, pp. 185-190, 2016

[34] V. Vapnik, Statistical Learning Theory, Wiley, 1998

[35] V. Vapnik, Estimation of Dependences Based on Empirical Data, Nauka, Springer Verlag, 2006
[36] B. E. Boser, I. V. V. Guyon, "A training algorithm for optimal margin classifiers", Fifth Annual Workshop on Computational Learning Theory, Pennsylvania, USA, July 27-29, 1992

[37] C. C. Chang, C. J. Lin, "LIBSVM: A library for support vector machines", ACM Transactions on Intelligent Systems and Technology, Vol. 2, No. 3, ArticleNo 27, 2011

[38] G. M. Foody, A. Mathur, "Toward intelligent training of supervised Image classifications: directing training data acquisition for SVM classification", Remote Sensing of Environment, Vol. 93, No. 1-2, pp. 107-117, 2004

[39] G. M. Foody, "Supervised image classification by MLP and RBF neural networks with and without an exhaustively defined set of classes", International, Journal of Remote Sensing, Vol. 25, No. 15, pp. 30913104, 2004

[40] G. I. J. Mountrakis, C. Ogole, "Support vector machines in remote sensing: A review", ISPRS Journal of Photogrammetry and Remote Sensing, Vol. 66, pp. 247-259, 2011

[41] B. Mulianga, A. Begue, P. Clouvel, P. Todoroff, "Mapping Cropping Practices of a Sugarcane-Based Cropping System in Kenya Using Remote Sensing”, Remote Sensing, Vol. 7, pp. 14428-14444, 2015

[42] Kenya Sugar Board, Year book of Statistics, KSB, 2012

[43] M. T. Blaschke, "Object based image analysis for remote sensing", ISPRS Journal of Photogrammetry and Remote Sensing, Vol. 65, pp. 216,2010

[44] D. C. Duro, S. E. Franklin, M. G. Dube, "A comparison of pixel-based and object-based Image analysis with selected machine learning algorithms for the classification of agricultural landscapes using SPOT-5 HRG imagery", Remote Sensing of Environment, Vol. 118, pp. 259-272, 2012

[45] J. Lowry, R. D. Ramsey, K. Thomas, D. Schrupp, T. Sajwaj, J. Kirby, E. Waller, S. Schrader, S. Falzarano, L. Langs, G. Manis, C. Wallace, K. Schulz, P. Comer, K. Pohs, W. Rieth, C. Velasquez, B. Wolk, W. Kepner, K. Boykin, L. O'Brien, D. Bradford, B. Thompson, J. PriorMagee, "Mapping Moderate-Scale Land-Cover over Very Large Geographic Areas within a Collaborative Framework: A Case Study of the Southwest Regional Gap Analysis Project (SWReGAP)", Remote Sensing of Environment, Vol. 108, pp. 59-73, 2007

[46] O. Nevalainen, E. Honkavaara, S. Tuominen, N. Viljanen, T. Hakala, X. Yu, J. Hyyppa, H. Saari, I. Polonen, N. N. Imai, A. M. G. Tommaselli, "Individual Tree Detection and Classification with UAV-Based Photogrammetric Point Clouds and Hyperspectral Imaging”, Remote Sensing Vol. 9, No. 3, ArticleNo 185, 2017

[47] N. Ekhtari, M. J. V. Zoej, M. R. Sahebi, A. Mohammadzadeh, "Automatic building extraction from LIDAR digital elevation models and World View imagery", Journal of Applied Remote Sensing. Vol. 3, No. 1, p. 033571, 2009

[48] L. Zhu, H. Shimamura, K. Tachibana, Y. Li, P. Gong, "Building Change Detection Based on Object Extraction in Dense Urban Areas", The International Archives of the Photogrammetry, Remote Sensing and Spatial Information Sciences Vol. 27B, pp. 905-908, 2008

[49] M. A. Friedl, D. K. McIver, J. C. F. Hodges, X. Y. Zhang, D. Muchoney, A. H. Strahler, C. E. Woodcock, S. Gopal, A. Schneider, A. Cooper, A. Baccini, F. Gao, C. Schaaf, "Global land cover mapping from MODIS: algorithms and early results", Remote Sensing of Environment, Vol. 83, No. 1-2, pp. 287-302, 2002

[50] J. P. M. O'Neil-Dunne, S. W. MacFaden, A. R. Royar, K. C. Pelletier, "An object-based system for LiDAR data fusion and feature extraction", Geocarto International, Vol. 28, No. 3, pp. 227-242, 2013

[51] T. M. Lillesand, R. W. Kiefer, J. Chipman, Remote Sensing and Image Interpretation, John Wiley \& Sons, 2004 